study BI 705564 was administered to 50 male $\mathrm{HV}$ at doses from 10 to $80 \mathrm{mg}$ once daily for 14 days. Blood samples were analyzed for BI 705564 plasma concentrations, BTK target occupancy (TO) and CD69 expression in B cells stimulated ex-vivo with anti-IgD.

Results All doses of BI 705564 were well-tolerated in both studies. There were no serious adverse events (AEs) and reported AEs were mainly of mild intensity and not doselimiting. There was no difference in the total number of subjects with AEs (combined data from both trials) between BI 705564 [28/75 (37.3\%)] and placebo [8/18 (44.4\%)] and no dose-relationship of AEs. The most frequently reported AE was headache (BI 705564: 8.0\% vs placebo: 5.6\%, combined data). There was no difference in the occurrence of infections (BI 705564: 6.5\% vs placebo: 11.1\%) or gastrointestinal disorders (BI 705564: $13.3 \%$ vs. placebo 11.1\%) in both studies (combined data). There were no relevant drug-related changes in vital signs, ECGs and standard safety laboratory tests. Analysis of BI 705564 plasma exposures showed a terminal half-life between 5.7 and 14.2 hour with no relevant accumulation after multiple dosing. Doses of $20 \mathrm{mg}$ and above resulted in an average TO $85 \%$ which is expected to result in clinical efficacy based on preclinical studies. After a single dose administration, TO was maintained for at least 48 hour consistent with the mechanism of a covalent irreversible inhibitor. Functional effects on BTK signalling were demonstrated by dose-dependent inhibition up to $100 \%$ of CD69 expression on anti-IgD stimulated B cells.

Conclusions BI 705564 was well-tolerated in healthy subjects after single and multiple doses. The favorable clinical safety profile and the high potential based on pre-clinical studies and effects on CD69 support further investigation of BI 705564 in patients with autoimmune diseases like SLE.

Funding Source(s): This study was funded by Boehringer Ingelheim.

\section{COMPOSITE GOALS PLUS INFLAMMATION: FURTHER RISK ASSESSMENT FOR SYSTEMIC LUPUS ERYTHEMATOSUS ASSOCIATED PULMONARY ARTERIAL HYPERTENSION IN CSTAR-PAH COHORT}

${ }^{1}$ Junyan Qian* , ${ }^{1}$ Mengtao Li, 'Q Qian Wang, 'Jiuliang Zhao, ${ }^{2}$ Zhuang Tian, ${ }^{1}$ Xiaofeng Zeng. ${ }^{1}$ Department of Rheumatology, Peking Union Medical College Hospital, Peking Union Medical College and Chinese Academy of Medical Science, Key Laboratory of Rheumatology and Clinical Immunology, Ministry of Education, Beijing, China; 'Department of Cardiology, Peking Union Medical College Hospital, Peking Union Medical College and Chinese Academy of Medical Science, Beijing, China

10.1136/lupus-2019-Ism.33

Background Chinese SLE treatment and Research Group (CSTAR) started a multi-center prospective cohort study recruiting SLE patients with pulmonary arterial hypertension (PAH) since 2006. This study aimed to investigate the validity of a multimensional risk assessment and the prognostic value of it in SLE-associated PAH.

Methods All SLE patients were fulfilled the 1997 revised ACR criteria. PAH was diagnosed based on ESC/ERS guidelines by right heart catheterization. The outcome was allcause mortality. Two different methods of risk categorization were applied according to baseline data, including low-risk criteria number of none to four and mean score of 1 (low- risk), 2 (intermediate-risk) or 3 (high-risk). According to first follow-up, patients were further divided into increased risk, remained risk and decreased risk group. A prediction model was used to distinguish SLE-PAH from vasculitic and vasculopathic subtype, based on the time interval between the diagnosis of SLE and PAH and Systemic Lupus Erythematosus Disease Activity Index (SLEDAI). KaplanMeier survival curves and Cox proportional hazards analysis were conducted.

Results 282 patients were enrolled. The 5 year survival of patients with none, one, two, three and four low risk criteria were $42.7 \%, 64.8 \%, 86.1 \%, 90.2 \%$ and $91.7 \%$, respectively $(\mathrm{HR}=0.59,95 \%$ CI $0.44-0.78, \mathrm{p}<0.001)$. The 5 year survival of patients in low-risk, intermediate-risk and highrisk group were $92.3 \%, 60.4 \%$ and $50.0 \%$, respectively (Log-rank, $\mathrm{p}=0.001)$. Notably, in low-risk group, patients with vasculitic subtype had better survival than those with vasculopathic subtype (Log-rank, $\mathrm{p}=0.044)$. The 5 year survival of patients with remained, decreased and increased risk were $65.4 \%, 88.1 \%$ and $23.8 \%$, respectively (log-rank, $\mathrm{p}<0.001$ ).

Conclusions Our study, for the first time, validated the prognostic value of risk stratification strategy at baseline and follow-up visit in patients with SLE-associated PAH. Patients are recommended to have a comprehensive evaluation on $\mathrm{PAH}$ and SLE at baseline and every follow-up visit. The SLE disease activity and systemic manifestations predict the phenotype of SLE-associated PAH, which also effect the long-term survival and need to be involved into risk assessment of SLEassociated PAH. Improving to low-risk group can be a future treatment target for SLE-associated PAH patients in clinical practice.

Funding Source(s): This work was supported by the Chinese National Key Research R and D Program [grant number 2017YFC0907601， 2017YFC0907602， 2017YFC0907603, 2008BAI59B02], and the Chinese National High Technology Research and Development Program, Ministry of Science and Technology [grant number 2012AA02A513].

\section{EVALUATION OF ADVERSE EVENTS AND RELAPSE RISK OF SYSTEMIC LUPUS ERYTHEMATOSUS DURING TREATMENT WITH HYDROXYCHLOROQUINE}

${ }^{1}$ Kunihiro Ichinose*, ${ }^{2}$ Keita Fujikawa, ${ }^{2}$ Akinari Mizokami, ${ }^{3}$ Toshiyuki Aramaki, ${ }^{3}$ Yukitaka Ueki, ${ }^{4}$ Momoko Okamoto, ${ }^{5}$ Toshimasa Shimizu, ${ }^{5}$ Tomohiro Koga, ${ }^{5}$ Shinya Kawashiri, ${ }^{5}$ Naoki Iwamoto, ${ }^{5}$ Takashi Igawa, ${ }^{5}$ Mami Tamai, ${ }^{5}$ Hideki Nakamura, ${ }^{6}$ Tomoki Origuchi, ${ }^{5}$ Atsushi Kawakami. 'Department of Immunology and Rheumatology, Advanced Preventive Medical Sciences, Graduate School of Biomedical Sciences, Nagasaki University; ${ }^{2}$ Department of Rheumatology, JCHO Isahaya General Hospital; ${ }^{3}$ Rheumatic disease center, Sasebo Chuo Hospital; ${ }^{4}$ Department of Immunology and Rheumatology, Unit of Advanced Preventive Medical Sciences, Nagasaki University Graduate School of Biomedical Sciences, Nagasaki, Japan; ${ }^{5}$ Department of Immunology and Rheumatology, Unit of Advanced Preventive Medical Sciences, Division of Advanced Preventive Medical Sciences, Nagasaki University Graduate School of Biomedical Sciences; ${ }^{6}$ Department of Rehabilitation Sciences, Nagasaki University Graduate School of Biomedical Sciences

\subsection{6/lupus-2019-Ism.34}

Background The antimalarial drug hydroxychloroquine (HCQ) has been widely used in the world to control the disease activity of systemic lupus erythematosus(SLE). In Japan, it has not been approved until September 2015 due to the problem of retinopathy induced by chloroquine. For these reasons, there is insufficient evidence for its effects and adverse events. 
Abstract 34 Table 1 The baseline characteristics of the patients $(n=109)$

\begin{tabular}{|c|c|c|c|c|c|}
\hline variables & median & IQR & variables & median & IQR \\
\hline Age at HCQ introduction, years & 40 & $(30.5-50.0)$ & Anti-ds-DNA antibodies, $\mathrm{U} / \mathrm{ml}$ & 8.2 & $(2.9-24.7)$ \\
\hline Gender (\%female) & $96 / 109(88.1)$ & Anti-RNP antibodies, U/ml & 16.5 & $(3.1-85.7)$ & \\
\hline Disease duration, months & 95 & $(38.0-184.5)$ & Anti-Sm antibodies, $\mathrm{U} / \mathrm{ml}$ & 4.5 & $(1.0-14.0)$ \\
\hline Body Mass Index(BMI) & 21 & $(18.9-23.9)$ & Anti-Ro/SSA antibodies, U/ml & 73.2 & $(4.2-126.5)$ \\
\hline Skin eruption & $53 / 109(48.6)$ & Anti-La/SSB antibodies, U/ml & 1.4 & $(0.7-5.0)$ & \\
\hline alopecia & $24 / 109(22.0)$ & $\mathrm{lgG}, \mathrm{mg} / \mathrm{dl}$ & 1403 & $(1135-1841)$ & \\
\hline arthralgia & $44 / 109$ (40.4) & $\lg A, \mathrm{mg} / \mathrm{dl}$ & 261 & $(203-355)$ & \\
\hline pregnancy & $4 / 109(3.7)$ & $\lg M, m g / d l$ & 99 & $(58.0-144.75)$ & \\
\hline White blood cell count, $/ \mu \mathrm{l}$ & 5500 & $(4245-7185)$ & $\mathrm{CH} 50, \mathrm{U} / \mathrm{ml}$ & 33 & $(25.6-43.6)$ \\
\hline 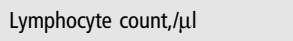 & 1099 & (713-1099) & $\mathrm{C} 3, \mathrm{mg} / \mathrm{dl}$ & 76.1 & $(61.3-88.3)$ \\
\hline Hemoglobin, g/dl & 11.9 & $(10.9-13.3)$ & $\mathrm{C} 4, \mathrm{mg} / \mathrm{dl}$ & 12.1 & $(8.7-17.8)$ \\
\hline Platelet counts, $\times 10^{4} / \mu \mathrm{l}$ & 21.9 & $(18.5-27.0)$ & SELENA-SLEDAI & 5 & $(3-8)$ \\
\hline Albumin, g/dl & 4 & $(3.7-4.3)$ & Comorbidities of SS (\%) & $28 / 109(25.7)$ & \\
\hline BUN, mg/dl & 13 & $(10.1-17.3)$ & Comorbidities of APS (\%) & $22 / 109(20.2)$ & \\
\hline $\mathrm{Cr}, \mathrm{mg} / \mathrm{dl}$ & 0.67 & $(0.57-0.76)$ & Comorbidities of LN (\%) & $32 / 109(29.4)$ & \\
\hline eGFR, $\mathrm{ml} / \mathrm{min} / 1.73 \mathrm{~m}^{2}$ & 81.2 & $(66.3-102.3)$ & Comorbidities of NPSLE (\%) & $21 / 109$ (19.3) & \\
\hline ANA & 320 & $(160-800)$ & & & \\
\hline
\end{tabular}

Here, we evaluated adverse events and relapse risk of SLE during the treatment of HCQ.

Methods We conducted an analysis of retrospectively collected data of 109 patients who were diagnosed as SLE and treated with HCQ at least 12 months at Nagasaki University Hospital and community hospitals. Demographic data included the patient's age at the onset of SLE, gender, the disease duration of SLE (the time from the diagnosis of SLE until the renal biopsy), comorbidities of Sjögren syndrome (SS)/anti-phospholipid syndrome (APS), and the treatment for induction. We identified the risk of adverse events and relapse at 12 months after introduction of HCQ.

Results Most of the patients were female (88.1\%). The median age at introduction of HCQ was 40.0 years (interquartile range [IQR] 30.550 .0 years), and the disease duration of SLE was 95 months (IQR 38.0184.5 months). The mean observation period after HCQ introduction was 12 months. The comorbidity rates of SS and APS were $25.7 \%$ and 20.2\%, respectively. The SELENA-SLEDAI decreased significantly after 3 months of introduction. The dose of oral prednisolone also decreased significantly after 6 months of introduction. Eighty-six cases $(78.9 \%)$ continued HCQ at 12 months after introduction. The adverse events appeared in 27 cases $(24.8 \%)$, including 11 cases of skin rashes $(10.1 \%)$ and 6 cases of gastrointestinal symptoms $(5.5 \%)$. Predictive factor for adverse event was white blood cell (WBC) counts at baseline (odds ratio: 0.9997, 95\% CI: $0.9994-0.9999, \mathrm{p}=0.0285)$. Twelve of 86 cases $(14.0 \%)$ experienced relapse those who needs to start prednisolone/ immunosuppressants or increase the dose of prednisolone. The multivariate analysis revealed $\mathrm{C} 4$ value at baseline was the predictive factor of relapse (odds ratio: $0.841,95 \% \mathrm{CI}$ : 0.718-0.984, $\mathrm{p}=0.0097)$.

Conclusions We retrospectively analyzed the risk of adverse events and relapse after HCQ introduction with a mean 12 month follow-up in SLE. The lower value of C4 at HCQ introduction was a predictive factor for relapse and the lower counts of WBC was a predictive factor for adverse event.

Funding Source(s): N/A

\section{PNEUMOCOCCAL VACCINATION AMONG IMMUNOSUPPRESSED LUPUS PATIENTS: WHO VACCINATES?}

${ }^{1}$ Shillpa Arora*, ${ }^{2}$ Ailda Nika, ${ }^{2}$ Winston Sequeira, ${ }^{2}$ Joel Block, ${ }^{2}$ Meenakshi Jolly. ${ }^{1}$ John H Jr Stroger Hospital of Cook County; ${ }^{2}$ Rush University Medical Center

\subsection{6/lupus-2019-Ism.35}

Background Center for Disease control and US department of Health and Human Services recommend Pneumococcal vaccination (PV) for chronic diseases and immunosuppressed. PV is a known quality indicator for patients with Systemic Lupus Erythematosus (SLE). We aimed to study the prevalence rates and predictors of PV in a cohort of SLE patients on immunosuppressive medications (ISM).

Methods Data were obtained through self-report questionnaires and medical chart review of 150 patients with SLE seen in rheumatology clinic at an academic center. Ninety four patients were eligible for PV based on ISM use (current use of any ISM other than hydroxychloroquine or current prednisone $7.5 \mathrm{mg}$ daily for 3 months or age 65 years). Information on rheumatologist recommendation/receipt of PV 23 in the preceding 5 years was collected by self-report in the questionnaire and/or from chart review. Information was also collected on demographics (age, gender, race, education, insurance), physician (having a primary care physician (PCP), the volume of SLE patients seen by each rheumatologist in the past 4 months) and severity and duration of SLE (ACR classification criteria, disease activity, damage, medications). Rheumatologists SLE volume was categorized as $0-50,50-100$ and $>100$. Univariate and Stepwise Binary logistic regression analysis were done to study predictors and most parsimonious model for PV (dependent variable).

Results Mean (SD) age of the cohort was 45.9 (15.0) years, 90\% were women: 52\% African-American, 26\% Caucasian, $7 \%$ Hispanic, $13 \%$ Asian and 2\% other. Mean (SD) SELENASLEDAI and SDI were 4.1 (2.3) and 1.7 (0.9), respectively. Over $90 \%$ had a PCP. SLE patient volume of rheumatologist during the previous 4 months was: $21 \%$ had $0-50,27 \%$ had $51-75$, while $52 \%$ had $>76$ SLE patients. Sixty-five $(69.1 \%)$ 